\title{
Simple Stochastic Games, Parity Games, Mean Payoff Games and Discounted Payoff Games are all LP-type Problems*
}

\author{
Nir Halman - MIT
}

Problem description: A Simple Stochastic Game (SSG) is defined on a directed graph with three types of vertices, min, max, and average, along with two sink vertices, the 0 -sink and the 1 -sink. The sink vertices have no outgoing edges. For every average vertex $a_{k}$, the outgoing edges from $a_{k}$ have positive rational weights such that the sum of their weights is 1 . The outgoing edges from the min and max vertices are unweighed. One of the vertices is a start vertex.

The game is a contest between two players, 0 and 1 . It is played in the following way. Begin by placing a token on the start vertex. When the token is on a min vertex $y_{j}$, player 0 moves it along one of the outgoing edges of $y_{j}$. When the token is on a max vertex $x_{i}$, player 1 moves it along one of the outgoing edges of $x_{i}$. When the token is on an average vertex $a_{k}$, the edge along which the token is moved is determined randomly, in proportion to the weights of the edges outgoing from $a_{k}$. The game ends when one of the sink vertices is reached. The goal of player 1 is to reach the 1 -sink. The goal of player 0 is to avoid the 1 -sink.

Before the game begins, player 1 chooses an outgoing edge from each max vertex. These selected edges will define a strategy for player 1 . During the game, whenever the token is on a max vertex, player 1 will move the token along the edge that is included in his strategy. Similarly, a choice of an outgoing edge for each min vertex is a strategy for player 0. Given a SSG, we would like to find the best strategies for both players. The corresponding decision problem is to determine whether player 1 wins with probability greater than $\frac{1}{2}$ when both players use their "best strategies". A special case of SSG's is a SSG which is defined on a directed graph where the outdegree of each vertex is at most two, and the weights of edges outgoing from average vertices are all $\frac{1}{2}$. Such a SSG is called a binary SSG.

*This work is based upon a chapter in the author's Ph.D. dissertation, prepared at Tel-Aviv University under the supervision of Professor Arie Tamir, and was published in Algorithmica, volume 49 (September 2007), pages 37-50.

†e-mail: halman@mit.edu 
Condon was the first to study SSG's from a complexity theory point of view. She showed in 1992 that the SSG decision problem is in NP $\cap$ co$\mathrm{NP}$ and hence is unlikely to be NP-complete, but at the same time it is not known to be in $\mathrm{P}$, despite substantial effort of the community. The first subexponential algorithm for binary SSG decision problem was an $e^{O(\sqrt{n})}$ time ad-hoc algorithm obtained in 1995 by Ludwig, for games with $n$ vertices.

SSG's are a restriction of stochastic games introduced by Shapley, some 50 years ago. Many variants of SSG's have been studied since then, e.g., Parity Games (PG's), Mean Payoff Games (MPG's), and Discounted Payoff Games (DPG's). SSG's are a generalization of PG's, MPG's and DPG's in the sense that there exists a polynomial time reduction from them to (nonbinary) SSG's. The decision problems corresponding to PG's, MPG's and DPG's are also known to be in NP $\cap$ co-NP. No polynomial time algorithm for any of the decision problems is yet known. The best known algorithm for PG's is the strongly subexponential ad-hoc algorithm of Björklund et al from 2003. The currently best algorithm for MPG's is the (not strongly) subexponential ad-hoc algorithm of Björklund et al from 2004. As far as we aware of, no subxponential algorithm for DPG's is known.

The technique used: In the early 90's Sharir and Welzl defined a model which generalizes Linear Programming (LP) and called it the LP-type model. An LP-type problem of combinatorial dimension $d$, where $d$ is independent of the size $n$ of the problem, is called fixed dimensional. Several algorithms that solve LP-type problems in time linear in $n$ are known, such as the ones of Clarkson, Sharir and Welzl, and Kalai. These algorithms run in time subexponential in $d$. By formulating problems as fixed-dimensional LP-type problems, and using the LP-type algorithms, one can obtain linear time algorithms to various optimization problems, mainly in computational geometry and location theory.

Our contribution: In this paper we demonstrate the applicability of the LP-type framework to "non-geometric" optimization problems. The main result of this paper is a formulation of a Simple Stochastic Game as a variabledimensional LP-type problem. Using this formulation, and the known algorithm of Sharir and Welzl for LP-type problems, we obtain the first strongly subexponential solution for SSG's (a strongly subexponential algorithm has only been known for binary SSG's). By applying known reductions between various games, we achieve the first strongly subexponential solution for DPG's and MPG's. We also give alternate simple proofs for the best known upper bounds for Parity Games and binary SSG's. While Ludwig and Björklund et al developed ad-hoc algorithms for each of the specific games they studied, we use only one unifying algorithm for all of these five games - the LP-type algorithm of Sharir and Welzl. 